\title{
DAOA/G72 predicts the progression of prodromal syndromes to first episode psychosis
}

\author{
Rainald Mössner · Anna Schuhmacher · Michael Wagner · Boris B. Quednow · Ingo Frommann · \\ Kai-Uwe Kühn · Sibylle G. Schwab · Marcella Rietschel · Peter Falkai · Wolfgang Wölwer · \\ Stephan Ruhrmann · Andreas Bechdolf · Wolfgang Gaebel · Joachim Klosterkötter · Wolfgang Maier
}

Received: 29 January 2009/Accepted: 25 August 2009/Published online: 10 September 2009

(C) Springer-Verlag 2009

\begin{abstract}
The genetic factors determining the progression of prodromal syndromes to first episode schizophrenia have remained enigmatic to date. In a unique prospective multicentre trial, we assessed whether variants at the D-amino acid oxidase activator (DAOA)/G72 locus influence progression to psychosis. Young subjects with a prodromal syndrome were observed prospectively for up to 2 years to assess the incidence of progression to schizophrenia or first
\end{abstract}

R. Mössner $(\bowtie) \cdot$ A. Schuhmacher · M. Wagner .

B. B. Quednow · I. Frommann · K.-U. Kühn ·

S. G. Schwab · W. Maier

Department of Psychiatry and Psychotherapy,

University of Bonn, Sigmund-Freud-Strasse 25,

53105 Bonn, Germany

e-mail: rainald.moessner@ukb.uni-bonn.de

B. B. Quednow

University Hospital of Psychiatry, University of Zurich,

Zurich, Switzerland

S. G. Schwab

Western Australian Institute for Medical Research and Centre for Medical Research, School of Psychiatry and Clinical Neurosciences and School of Medicine and Pharmacology,

University of Western Australia, Nedlands, Australia

M. Rietschel

Central Institute of Mental Health, Mannheim, Germany

P. Falkai

Department of Psychiatry and Psychotherapy,

University of Göttingen, Göttingen, Germany

W. Wölwer · W. Gaebel

Department of Psychiatry and Psychotherapy,

University of Düsseldorf, Düsseldorf, Germany

S. Ruhrmann · A. Bechdolf · J. Klosterkötter Department of Psychiatry and Psychotherapy, University of Cologne, Cologne, Germany episode psychosis. Of the 82 probands with a prodromal syndrome, 21 probands experienced progression to psychosis within the observation period. Assessment of nine common variants in the DAOA/G72 locus yielded two variants with the predictive value for symptom progression: all four probands with the rs 1341402 CC genotype developed psychosis compared with 17 out of 78 probands with the TT or CT genotypes $\left(\chi^{2}=12.348 ; d f=2\right.$; $p=0.002$ ). The relative risk for progression to psychosis was significantly increased in the $\mathrm{CC}$ genotype: $\mathrm{RR}=4.588 \quad(95 \% \quad \mathrm{CI}=2.175-4.588) . \quad$ Similarly, for rs $778294,50 \%$ of probands with the AA genotype, but only $22 \%$ of probands with a GG or GA genotype progressed to psychosis $\left(\chi^{2}=7.027 ; d f=2 ; p=0.030\right)$. Moreover, haplotype analysis revealed a susceptibility haplotype for progression to psychosis. This is one of the first studies to identify a specific genetic factor for the progression of prodromal syndromes to schizophrenia, and further underscores the importance of the DAOA/G72 gene for schizophrenia.

Keywords Schizophrenia - Prodromal · Progression . D-Amino acid oxidase activator $\cdot$ G72 gene

\section{Introduction}

The development of a first episode of schizophrenia is often preceded by prodromal states with variable symptoms [22]. The factors determining the progression of disease from a prodromal state to a psychotic episode are of great interest [3]. However, little is currently known about the factors influencing whether a prodromal proband progresses to psychosis or remains stable [6]. We hypothesised that genetic variants play an important role in determining 
symptom progression. Amongst the schizophrenia susceptibility genes identified in the past few years [15], we focused on a well characterised schizophrenia susceptibility locus, DAOA/G72, and queried whether variants in this locus might influence this progression.

The DAOA/G72 gene was originally discovered during the analysis of the schizophrenia susceptibility region on chromosome 13. Fine-mapping of the region on the long arm of chromosome 13,13q12-13q34 led to the discovery of the new gene G72 [4]. Variants in this gene were related to schizophrenia in Canadian and Russian subjects. The G72 gene is transcribed in brain, and increased expression was found in the dorsolateral prefrontal cortex in schizophrenia compared with controls [12]. To assess the function of this gene, a screen of binding proteins was performed, which showed that G72 binds to and activates D-amino acid oxidase. Therefore, G72 was named D-amino acid oxidase activator (DAOA) [4]. DAOA/G72 is a very attractive schizophrenia candidate gene also due to the fact that it is a primate-specific gene [4]. A recent meta-analysis of DAOA/G72 in schizophrenia showed highly significant evidence of association between a number of markers in the DAOA/G72 region and schizophrenia [5]. These authors concluded that the association findings for the DAOA/G72 gene and schizophrenia are amongst the most compelling in psychiatry. This led us to focus on the DAOA/G72 gene, since we expected a sufficiently large effect to be visible in a prospective investigation.

The main outcome measure of the present study was progression to a psychosis, namely the incidences of first episode schizophrenia and first episode psychosis. In a unique prospective multicenter approach combining psychopathology and genetics, prodromal subjects were followed for up to 2 years to prospectively identify those probands who would progress to psychosis. Specifically, we assessed here whether common DAOA/G72 variants might predict the conversion to a psychosis in this prospective multicentre study. We analysed a number of variants which are located in the coding region of DAOA/ G72, but also variants in the $5^{\prime}$ and $3^{\prime}$ putative regulatory regions which are expected to have an important role in the function and expression of the DAOA/G72 gene.

\section{Materials and methods}

\section{Study subjects}

The clinical study was conducted at three early detection and intervention centres. The centres were located at the Departments of Psychiatry and Psychotherapy at the Universities of Bonn, Cologne, and Düsseldorf. To aid in the recruitment of probands with prodromal syndromes, awareness programmes were put in place for primary and psychiatric health services, for the relatives of patients with schizophrenia, and were also aimed to inform youth support services as well as the general population. The aims of the programmes were to establish local mental health networks as well as the facilitation for persons at a risk to find help with the early intervention services. Information was provided about early symptoms of schizophrenia as well as about the usefulness of early intervention. Referrals of probands were from a number of settings, including from counselling services, youth support services, primary health care, and mental health professionals [11].

The clinical study and the inclusion and exclusion criteria are described in more detail elsewhere [1]. Briefly, patients were eligible for inclusion in the trial if they met the criteria of the early or late initial prodromal state and did not fulfil exclusion criteria. The early initial prodromal state was operationally defined by either basic symptoms predicting psychosis [10] or by a clinically relevant decline of functioning in combination with established risk factors (risk and decreased functioning) [22]. Basic symptoms required the presence of one or more of the following symptoms several times per week during the last 3 months: thought interferences, thought perseverations, thought pressure, thought blockages, disturbances of receptive language, decreased ability to discriminate between ideas and perception or between fantasy and true memory contents, tendency to delusion of reference with subject centrism, derealisation, visual perception disturbances, and acoustic perception disturbances. Established risk factors were pre- or perinatal complications or a first-degree relative with a lifetime diagnosis of schizophrenia or a schizophrenia spectrum disorder. Late initial prodromal state was defined by the presence of at least one of the following symptoms appearing several times a week: ideas of reference, odd beliefs or magical thinking, unusual perceptual experiences, odd thinking and speech, or suspiciousness or paranoid ideation; or by the appearance of one of the following symptoms for less than 1 week and resolving spontaneously: hallucinations, delusions, formal thought disorder, or gross disorganised or catatonic behaviour. Probands were only included if aged between 17 and 42 years. Exclusion criteria of note were previous treatment with antipsychotics, present or past diagnosis of a schizophrenic, schizophreniform, schizoaffective, delusional, or bipolar disorder, present or past diagnosis of a brief psychotic disorder with a duration of more than 1 week or within the last 4 weeks regardless of its duration, organic brain disease, and alcohol or drug dependence within the last 3 months. During the study, probands were seen approximately once a month. Progression to first episode psychosis was operationally defined by cut-off points on positive and negative syndrome scale (PANSS) [8] subscales of 4 or more on hallucinations, 4 or more on 
delusions and 5 or more on conceptional disorganisation or formal thought disorder for at least 7 days and meeting criteria for schizophrenia (ICD-10: F20) or acute schizophrenia-like psychotic disorder (ICD-10: F23.2). The study was approved by the appropriate ethics committees.

\section{Genotyping}

Nine common variants spanning the DAOA/G72 gene were identified from the UCSC genome browser (genome. cse.ucsc.edu). Single-nucleotide polymorphism nomenclature is based on the NCBI dbSNP build 129 (April 2008, updated June 2008). Moreover, the traditional M nomenclature as described by Chumakov et al. [4] is given, whenever applicable. Venous blood was taken at study entry and DNA extracted by standard protocols. DAOA/G72 variants were genotyped by allele discrimination using TaqMan technology; $12.5 \mathrm{ng}$ of DNA was used for TaqMan SNP genotyping assays on demand, employing the Applied Biosystems protocol. Following the completion of PCR reactions, fluorescence was determined on a Tecan 384 Ultra reader (Tecan USA, Durham, NC). The probes were labelled with carboxyfluorescein (FAM), with an excitation wavelength of $485 \mathrm{~nm}$ and an emission wavelength of $535 \mathrm{~nm}$. VIC-labelled probes employed an excitation wavelength of $535 \mathrm{~nm}$ and an emission wavelength of $590 \mathrm{~nm}$.

\section{Statistical analysis}

The influence of DAOA/G72 variants on progression of symptoms was assessed by $\chi^{2}$ tests, employing SPSS version 14.0 (SPSS, Chicago, USA). If cells had an expected frequency of $<5$, Monte Carlo simulation based on 10,000 permutations with the start value $92,208,573$ was done. The level of significance was set at $p<0.05$ (two sided). To correct for multiple testing, the spectral decomposition method was employed to estimate the number of effective loci $[14,17]$. Age distribution of the proband groups was assessed by ANOVA. Haplotype block structure was determined by the solid spine of LD method as implemented in Haploview (Broad Institute, Mass, USA). Haplotype analysis was performed employing the program Famhap 17 [2]. Famhap 17 yields a global significance value of the haplotype analysis employing the likelihood ratio test, as well as individual $p$ values for each haplotype. Individual haplotype $p$ values were subsequently corrected for multiple testing employing Bonferroni correction.

\section{Results}

Eighty-two probands were enrolled in the prospective study of early prodromal states. They were followed longitudinally and were assessed for progression to psychosis. DNA was obtained for subsequent determination of DAOA/G72 variants. The mean age of the probands was $26.3 \pm 7.1$ years. Twenty-one probands experienced a progression of symptoms to schizophrenia or first episode psychosis. The mean duration for progression to psychosis was 29.7 weeks (range 2-86 weeks). The mean age of those patients progressing to psychosis was $24.2 \pm 5.0$ years and they were thus on an average slightly younger than those probands who remained stable $(27.0 \pm 7.6$ years; $F=3.139 ; p=0.083$ by Welch test). The gender distribution did not differ between the probands who progressed and those who remained stable $\left(\chi^{2}=1.320 ; d f=1\right.$; $p=0.251)$.

We then queried whether the likelihood of progression to psychosis is related to variants of the DAOA/G72 gene. Two variants were found to have an impact on the progression to psychosis. Thus, for rs 1341402, 20.4\% of probands with the TT genotype and $24.1 \%$ of heterozygous probands (TC genotype) progressed to psychosis (Table 1). In contrast, all homozygous CC probands progressed to psychosis. This effect was statistically significant $\left(\chi^{2}=12.348, d f=2, p=0.002\right)$. This finding remained significant after the correction for multiple testing with the spectral decomposition method [14, 17]: with an effective number of independent marker loci of 5 , the significance threshold is $p=0.01$. A weaker effect was seen for another DAOA/G72 variant, rs778294 (M19). For the AA genotype, $50 \%$ of probands progressed to psychosis (5 out of 10 probands with the AA genotype). In contrast, only $13.2 \%$ of probands with the GG genotype progressed. Heterozygous GA probands experienced a progression to psychosis in $32.4 \%$ of cases. The increased likelihood of progression for the AA genotype was nominally significant $\left(\chi^{2}=7.027, d f=2, p=0.030\right)$.

A linkage plot of the nine variants analysed in the 82 prodromal probands is shown in Fig. 1. As can be seen, the analysed markers segregate into two haplotype blocks. Markers rs1341402 and rs778294 are located in the same haplotype block 1 . Given a $\mathrm{D}^{\prime}$ of 0.95 between markers rs1341402 and rs778294, we constructed a two-marker haplotype comprising these two variants. Haplotype analysis of this two-marker haplotype revealed two haplotypes that had an impact on the progression of prodromal patients to psychosis (Table 2). Thus, haplotype $\mathrm{C}-\mathrm{A}$ is present in $35.7 \%$ of prodromal patients who progressed to psychosis, but only in $17.1 \%$ of prodromal subjects who remained stable ( $p=0.046$; Bonferroni corrected $p$ value). Conversely, haplotype $\mathrm{T}-\mathrm{G}$ is present in $72 \%$ of prodromal subjects who remained stable compared with $50 \%$ of prodromal patients who progressed to psychosis $(p=0.031$; Bonferroni corrected $p$ value). Thus, haplotype $\mathrm{C}-\mathrm{A}$ increases the susceptibility for progression to psychosis, 
Table 1 Progression of prodromal probands to psychosis stratified according to DAOA/G72 genotype

\begin{tabular}{|c|c|c|c|c|c|}
\hline \multirow{2}{*}{$\begin{array}{l}\text { Polymorphism } \\
\text { rs1935058 }\end{array}$} & \multicolumn{3}{|c|}{ Progression to psychosis according to genotype } & \multirow{2}{*}{$\frac{\chi^{2}}{2.182}$} & \multirow{2}{*}{$\frac{p \text { value }}{0.336}$} \\
\hline & TT: 9/26 (34.6\%) & TC: $7 / 38(18.4 \%)$ & CC: $5 / 18(27.8 \%)$ & & \\
\hline rs1341402 & TT: $10 / 49(20.4 \%)$ & TC: $7 / 29(24.1 \%)$ & CC: $4 / 4(100 \%)$ & 12.348 & $0.002 * *$ \\
\hline rs2391191 (M15) & GG: $11 / 34(32.4 \%)$ & GA: $7 / 36(19.4 \%)$ & AA: $3 / 12(25.0 \%)$ & 1.532 & 0.465 \\
\hline rs1935062 & AA: $12 / 39(30.8 \%)$ & AC: $6 / 30(20.0 \%)$ & CC: $3 / 13(23.1 \%)$ & 1.084 & 0.582 \\
\hline rs947267 (M18) & AA: $3 / 20(15 \%)$ & AC: $10 / 41(24.4 \%)$ & CC: $8 / 21(38.1 \%)$ & 2.932 & 0.231 \\
\hline rs778294 (M19) & GG: $5 / 38(13.2 \%)$ & GA: 11/34 (32.4\%) & AA: $\mathbf{5 / 1 0}(\mathbf{5 0 . 0 \%})$ & 7.027 & $0.030 *$ \\
\hline rs778293 (M22) & AA: $7 / 29(24.1 \%)$ & AG: $10 / 44(22.7 \%)$ & GG: 4/9 (44.4\%) & 1.901 & 0.387 \\
\hline rs3918342 (M23) & CC: $8 / 23(34.8 \%)$ & CT: $10 / 39(25.6 \%)$ & TT: $3 / 20(15.0 \%)$ & 2.198 & 0.333 \\
\hline rs1421292 (M24) & TT: 8/24 (33.3\%) & TA: $10 / 40(25.0 \%)$ & AA: $3 / 18(16.7 \%)$ & 1.515 & 0.469 \\
\hline
\end{tabular}

Probands progressing to psychosis/number of prodromal probands with a given genotype are shown

Single-nucleotide polymorphism nomenclature is based on the NCBI dbSNP build 129 (April 2008, updated June 2008)

The traditional $\mathrm{M}$ nomenclature as described by Chumakov et al. is also given, whenever applicable

Nominal $p$ values are shown. The nominal significance level is set at $p<0.05$ (the significance

Threshold is 0.01 after correction for multiple testing according to Nyholt 2004/Li and Ji 2005)

** $d f=2$; Monte Carlo simulation yielded a significance of $p=0.004$

$* d f=2$; Monte Carlo simulation yielded a significance of $p=0.035$

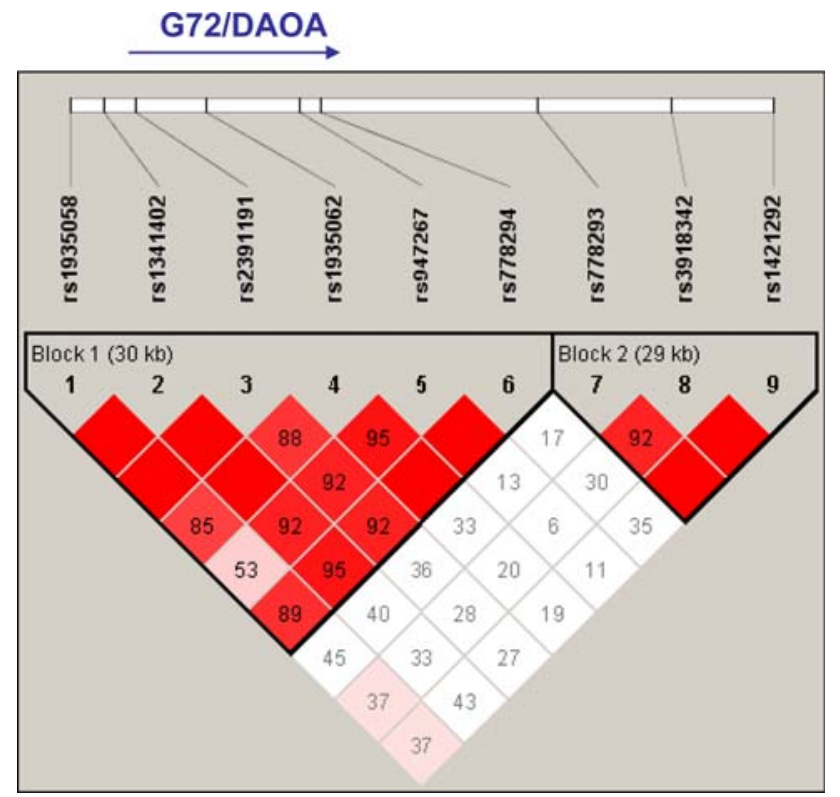

Fig. 1 Haplotype block structure of the nine analysed SNPs spanning the DAOA/G72 gene. The variants segregate into two haplotype blocks. Haplotype block structure was determined in the 82 prodromal subjects employing the solid spine of LD method as implemented in Haploview. The location of DAOA/G72 is also shown

while haplotype $\mathrm{T}-\mathrm{G}$ is protective. The global $p$ value for this haploype analysis was $p=0.047$.

When haplotype analysis was performed on all six markers contained in haplotype block 1 , no significant association of the six marker haplotypes, with progression to psychosis was seen ( $p=0.21$; likelihood ratio test). Similarly, haplotype analysis of the markers in block 2 was not significant $(p=0.295$; likelihood ratio test).
Next, we assessed whether the positive and negative syndrome scale (PANSS) scores at study entry were related to the long-term progression of the disorder. PANSS scores at study entry were available for 75 out of the 82 study probands. Positive symptom scores at study entry related with later progression: those probands who would progress during the study period had an initial score of 13.53 compared with 10.64 for those probands who remained stable $(F=9.030 ; p=0.004)$. Negative symptoms and total PANSS scores also showed an, albeit lesser, influence. Thus, probands with later progression had an initial negative symptom score of 15.0 compared with those who remained stable $(12.34 ; F=5.690 ; p=0.02)$. For total PANSS scores, the respective values were 60.37 (later progression) and 52.04 (stable probands; $F=6.196$; $p=0.015$ ). Global PANSS scores did not relate to progression.

Finally, we queried whether DAOA/G72 marker rs1341402, which had the greatest influence on progression, also had an influence on PANSS scores at study entry. We found that the probands with the CC genotype, all of whom later progressed to psychosis, had higher PANSS scores at study entry compared with the other genotypes (Table 3). For example, probands with the CC genotype had a positive PANSS score of 16.5 compared with 11.08 for the TT/CT genotypes $(p=0.025)$. A similar difference was found for negative symptoms: 17.25 versus 12.77 ( $p=0.033)$. Moreover, similar findings were seen in the haplotype analysis (Table 4). Thus, probands with two copies of the risk-haplotype $\mathrm{C}-\mathrm{A}$ had higher positive symptom scores at study entry $(16.50 ; p=0.014)$, and probands lacking the protective haplotype $\mathrm{T}-\mathrm{G}$ also had 
Table 2 Haplotype analysis of the two-marker haplotype rs1341402rs778294 of DAOA/G72 in prodromal subjects

\begin{tabular}{lllc}
\hline $\begin{array}{l}\text { Haplotype } \\
\text { (rs1341402-rs778294) }\end{array}$ & $\begin{array}{l}\text { Frequency } \\
\text { in patients } \\
\text { progressing to } \\
\text { psychosis (\%) }\end{array}$ & $\begin{array}{l}\text { Frequency } \\
\text { in probands } \\
\text { remaining } \\
\text { stable (\%) }\end{array}$ & $p$ value $^{\#}$ \\
\hline T-G & 50.0 & 72.0 & $\mathbf{0 . 0 3 1 *}$ \\
T-A & 14.3 & 9.93 & $>0.1$ \\
C-A & 35.7 & 17.1 & $\mathbf{0 . 0 4 6}^{*}$ \\
\hline
\end{tabular}

The global significance of this haplotype analysis was $p=0.047$ (likelihood ratio test)

\# $p$ values are Bonferroni corrected

* $p$ value significant after Bonferroni correction

Table 3 PANSS scores at study entry stratified according to DAOA/ G72 rs1341402 genotype

\begin{tabular}{lcll}
\hline PANSS scales & rs1341402 CC & rs1341402 TT/TC & $p$ value* \\
\hline Positive symptoms & $16.5 \pm 5.06$ & $11.08 \pm 3.55$ & $\mathbf{0 . 0 2 5}$ \\
Negative symptoms & $17.25 \pm 3.30$ & $12.77 \pm 4.27$ & $\mathbf{0 . 0 3 3}$ \\
Global PANSS score & $34.75 \pm 7.63$ & $29.47 \pm 7.03$ & 0.171 \\
Total PANSS score & $68.5 \pm 14.47$ & $53.33 \pm 12.58$ & $\mathbf{0 . 0 4 7}$ \\
\hline
\end{tabular}

* Mann-Whitney test with two-sided Monte Carlo significance. 10,000 simulations with a start value of $2,000,000$ were done

higher positive (14.56; $p=0.012$ ) and total (63.22; $p=0.03)$ PANSS scores at study entry compared with the other haplotypes (Table 4).

\section{Discussion}

This is one of the first prospective studies of prodromal subjects that assess the contribution of genetic variants to the risk of progression to psychosis. In a multicenter study, we have identified probands with a prodromal state and followed them longitudinally to assess the progression of symptoms.

Two variants of DAOA/G72 had an important influence on the progression of the prodromal subjects. Thus, all homozygous carriers of the C allele of rs1341402 experienced a progression of symptoms. Similarly, $50 \%$ of probands homozygous for the A allele of rs778294 (M19) had a progression of symptoms. Therefore, these variants of DAOA/G72 identify probands at a very high risk of progression, despite the overall risk of progression of the probands in the prodromal state being much lower: in the overall sample, 21 out of 82 study subjects experienced a transition, corresponding to $25.6 \%$ of prodromal subjects. The relative risk for progression to psychosis was significantly increased in homozygous carriers of the rs $1341402 \mathrm{C}$ allele ( $\mathrm{RR}=4.588,95 \% \mathrm{CI}=2.175-4.588, p=0.003$ ). The increase in relative risk for subjects homozygous for the rs778294 (M19) A allele failed to reach significance $(\mathrm{RR}=2.250,95 \% \mathrm{CI}=0.967-3.987, p=0.114)$. These results indicate that genetic variants in the DAOA gene may substantially potentiate the risk for subsequent psychosis in subjects with prodromal symptoms, and may thus aid in treatment decisions.

Our findings raise the question of the mechanism by which DAOA/G72 determines the increased risk for prodromal probands to develop schizophrenia. The DAOA/ G72 gene has since its discovery been described as the activator of D-amino acid oxidase (DAO) [4]. Given that this enzyme oxidises D-serine, which is an agonist at glutamatergic NMDA receptors, DAOA/G72 has been linked to the glutamate dysfunction hypothesis of schizophrenia. Very recently, G72 has also been linked to a neurodevelopmental role. Thus, G72 was found to promote dendritic branching in hippocampal neurons by way of expression in the mitochondria. This effect was seen only during the period of active dendritogenesis in synaptically immature neurons [13]. Moreover, the expression of G72 protein in the amygdala was confirmed [13]. It is currently not known which effect of the DAOA/G72 gene, namely the D-serine/glutamate pathway or the mitochondrial function pathway, is mainly responsible for the role of DAOA/G72 in schizophrenia. Both mechanisms could contribute to the structural and functional brain alterations that may precede the onset of schizophrenia $[16,18,21]$.

A meta-analysis of published association studies found highly significant associations between variants of DAOA/

Table 4 PANSS scores at study entry stratified according to DAOA/G72 two-marker haplotype rs1341402-rs778294

\begin{tabular}{|c|c|c|c|c|c|c|c|c|}
\hline \multirow[t]{2}{*}{ PANSS scales } & \multicolumn{3}{|l|}{ Haplotype $\mathrm{T}-\mathrm{G}$} & \multirow[t]{2}{*}{$p$ value } & \multicolumn{3}{|l|}{ Haplotype C-A } & \multirow[t]{2}{*}{$p$ value } \\
\hline & $0 \times$ & $1 \times$ & $2 \times$ & & $0 \times$ & $1 \times$ & $2 \times$ & \\
\hline Positive symptoms & $14.56 \pm 3.81$ & $10.39 \pm 3.41$ & $11.48 \pm 3.79$ & 0.012 & $11.35 \pm 3.77$ & $10.60 \pm 3.12$ & $16.50 \pm 3.81$ & 0.014 \\
\hline Negative symptoms & $15.11 \pm 4.78$ & $12.09 \pm 4.20$ & $13.36 \pm 4.22$ & 0.148 & $12.67 \pm 4.19$ & $12.96 \pm 4.51$ & $17.25 \pm 3.30$ & 0.128 \\
\hline Global PANSS score & $33.56 \pm 5.64$ & $28.21 \pm 7.56$ & $30.27 \pm 6.71$ & 0.116 & $29.43 \pm 7.12$ & $29.56 \pm 7.01$ & $34.75 \pm 7.63$ & 0.357 \\
\hline Total PANSS score & $63.22 \pm 12.18$ & $50.7 \pm 12.46$ & $55.12 \pm 12.83$ & 0.030 & $53.46 \pm 13.14$ & $53.12 \pm 11.76$ & $68.50 \pm 14.48$ & 0.075 \\
\hline
\end{tabular}

PANSS scores are given for probands with 0,1 or 2 copies of each haplotype

$p$ values were calculated by one-way ANOVA 
G72 and schizophrenia as well as less significant evidence of an association with bipolar disorder [5], although a very recent meta-analysis showed weaker effects [20]. The associated markers varied across studies. Thus, it remained unclear whether the associated markers all reflect the same functional variation or, more likely, multiple functionally important variants are present in a large region encompassing DAOA/G72 [5]. Notably, markers rs778293 (M22), rs3913842 (M23), and rs1421292 (M24) which we have previously shown to be associated with schizophrenia [19] show no linkage disequilibrium with the other markers analysed. This further suggests that there is more than one functional allele contributing to schizophrenia susceptibility in the DAOA/G72 region. Interestingly, these three markers are located more than $50 \mathrm{~kb}$ downstream of the coding region of DAOA/G72. The only other gene in this area is a hypothetical gene called G30, which is located on the opposite strand and might provide antisense regulation of the DAOA/G72 transcript. These three markers are, therefore, likely to exert effect independent from the other DAOA/G72 markers. In our study, these three markers did not influence progression of prodromal probands, in contrast to the markers located in the putative promotor region or in the coding region of DAOA/G72.

We then queried whether the variants relating to symptom progression of prodromal patients may be functional. The variant most strongly associated with the progression of prodromal subjects is rs1341402. This variant is located at position -2854 with respect to the transcription start site. It is, thus, located in the extended putative promotor region of G72. Because common promotor variants frequently have major influence on the expression level of the gene product, rs1341402 is an excellent functional candidate variant in the promotor region of G72. The second variant relating to symptom progression is rs778294 (M19). This variant is situated $15 \mathrm{bp}$ upstream of the $5^{\prime}$ end of a protein-coding exon of G72 [4]. As Chumakov et al. [4] note this variant is part of the consensus sequence of an acceptor splice site and thus could potentially alter the splicing of G72. This variant is in high linkage disequilibrium with the mostly associated variant rs1341402, with a $\mathrm{D}^{\prime}$ value of 0.95 (Fig. 1). A variant that does not relate to symptom progression of prodromal subjects is rs2391191 (M15). This is a coding variant of G72, leading to a lysine/arginine exchange. Because this is a conservative amino acid exchange, it is not surprising that this variant does not relate to symptom progression in prodromal subjects.

Progression of prodromal probands to schizophrenia has previously been investigated with regard to the neuregulin 1 (NRG1) gene. An NRG1 variant was related to progression to schizophrenia in prodromal probands [9]. The same variant was implicated in the development of psychosis in high-risk subjects for psychosis [7]. The sample size of the study group in these two studies was similar to the present study; with 67 prodromal probands [9] and 79 high-risk subjects [7] investigated, respectively, compared with 82 prodromal probands in the present study.

In conclusion, DAOA/G72 is one of the first genes identified to influence the risk of symptom progression of schizophrenia prodromal probands. Variation in DAOA/ G72 identified here explains part of the risk of progression. We anticipate that other genes acting either alone or in concert with DAOA/G72 and NRG1 will be identified in the future to also influence the risk of progression to schizophrenia. These other genes may act in the same pathophysiological pathways as DAOA/G72, or operate in different pathogenic biochemical pathways. Thus, it may be feasible in the future to establish a risk profile of prodromal probands according to their combination of risk alleles and identify those probands in whom a prophylactic treatment with neuroleptics or other as yet to be discovered treatment modalities should be instituted immediately to prevent progression to schizophrenia.

Acknowledgments We thank all prodromal probands for their participation in this study. We thank V. Guttenthaler, A. Lentzen, and C. Hanses for expert technical assistance. This study was part of the German Research Network on Schizophrenia and was funded by the German Federal Ministry for Education and Research BMBF (grants 01GI0501, 01GI0232 and 01GI0234). B. B. Quednow was supported by the Deutsche Forschungsgemeinschaft (DFG, grant QU 218/1-1) and by the Nachwuchsförderungskredit of the University of Zürich.

\section{References}

1. Bechdolf A, Ruhrmann S, Wagner M, Kühn KU, Janssen B, Bottlender R, Wieneke A, Schulze-Lutter F, Maier W, Klosterkötter J (2005) Interventions in the initial prodromal states of psychosis in Germany: concept and recruitment. Br J Psychiatry Suppl 48:s45-s48

2. Becker T, Knapp M (2004) A powerful strategy to account for multiple testing in the context of haplotype analysis. Am J Hum Genet 75:561-570

3. Bogren M, Mattisson C, Tambs K, Horstmann V, MunkJorgensen P, Nettelbladt P (2009) Predictors of psychosis: a 50-year follow-up of the Lundby population. Eur Arch Psychiatry Clin Neurosci

4. Chumakov I, Blumenfeld M, Guerassimenko O, Cavarec L, Palicio M, Abderrahim H, Bougueleret L, Barry C, Tanaka H, La Rosa P, Puech A, Tahri N, Cohen-Akenine A, Delabrosse S, Lissarrague S, Picard FP, Maurice K, Essioux L, Millasseau P, Grel P, Debailleul V, Simon AM, Caterina D, Dufaure I, Malekzadeh K, Belova M, Luan JJ, Bouillot M, Sambucy JL, Primas G, Saumier M, Boubkiri N, Martin-Saumier S, Nasroune M, Peixoto H, Delaye A, Pinchot V, Bastucci M, Guillou S, Chevillon M, Sainz-Fuertes R, Meguenni S, Aurich-Costa J, Cherif D, Gimalac A, Van Duijn C, Gauvreau D, Ouellette G, Fortier I, Raelson J, Sherbatich T, Riazanskaia N, Rogaev E, Raeymaekers P, Aerssens J, Konings F, Luyten W, Macciardi F, Sham PC, Straub RE, Weinberger DR, Cohen N, Cohen D (2002) Genetic and physiological data implicating the new human gene 
G72 and the gene for D-amino acid oxidase in schizophrenia. Proc Natl Acad Sci USA 99:13675-13680

5. Detera-Wadleigh SD, McMahon FJ (2006) G72/G30 in schizophrenia and bipolar disorder: review and meta-analysis. Biol Psychiatry 60:106-114

6. Gschwandtner U, Pflueger MO, Semenin V, Gaggiotti M, Riecher-Rossler A, Fuhr P (2009) EEG: a helpful tool in the prediction of psychosis. Eur Arch Psychiatry Clin Neurosci 259:257-262

7. Hall J, Whalley HC, Job DE, Baig BJ, McIntosh AM, Evans KL, Thomson PA, Porteous DJ, Cunningham-Owens DG, Johnstone EC, Lawrie SM (2006) A neuregulin 1 variant associated with abnormal cortical function and psychotic symptoms. Nat Neurosci 9:1477-1478

8. Kay SR, Fiszbein A, Opler LA (1987) The positive and negative syndrome scale (PANSS) for schizophrenia. Schizophr Bull $13: 261-276$

9. Keri S, Kiss I, Kelemen O (2009) Effects of a neuregulin 1 variant on conversion to schizophrenia and schizophreniform disorder in people at high risk for psychosis. Mol Psychiatry 14:118-119

10. Klosterkötter J, Hellmich M, Steinmeyer EM, Schultze-Lutter F (2001) Diagnosing schizophrenia in the initial prodromal phase. Arch Gen Psychiatry 58:158-164

11. Köhn D, Berning J, Maier W (2002) German awareness program for early detection and early intervention. Acta Psychiatrica Scandinavica 106:s19

12. Korostishevsky M, Kaganovich M, Cholostoy A, Ashkenazi M, Ratner Y, Dahary D, Bernstein J, Bening-Abu-Shach U, Ben-Asher E, Lancet D, Ritsner M, Navon R (2004) Is the G72/ G30 locus associated with schizophrenia? Single nucleotide polymorphisms, haplotypes, and gene expression analysis. Biol Psychiatry 56:169-176

13. Kvajo M, Dhilla A, Swor DE, Karayiorgou M, Gogos JA (2008) Evidence implicating the candidate schizophrenia/bipolar disorder susceptibility gene G72 in mitochondrial function. Mol Psychiatry 13:685-696
14. Li J, Ji L (2005) Adjusting multiple testing in multilocus analyses using the eigenvalues of a correlation matrix. Heredity 95:221227

15. Maier W (2008) Common risk genes for affective and schizophrenic psychoses. Eur Arch Psychiatry Clin Neurosci 258(Suppl 2):37-40

16. Morey RA, Inan S, Mitchell TV, Perkins DO, Lieberman JA, Belger A (2005) Imaging frontostriatal function in ultra-highrisk, early, and chronic schizophrenia during executive processing. Arch Gen Psychiatry 62:254-262

17. Nyholt DR (2004) A simple correction for multiple testing for single-nucleotide polymorphisms in linkage disequilibrium with each other. Am J Hum Genet 74:765-769

18. Pantelis C, Velakoulis D, McGorry PD, Wood SJ, Suckling J, Phillips LJ, Yung AR, Bullmore ET, Brewer W, Soulsby B, Desmond P, McGuire PK (2003) Neuroanatomical abnormalities before and after onset of psychosis: a cross-sectional and longitudinal MRI comparison. Lancet 361:281-288

19. Schumacher J, Jamra RA, Freudenberg J, Becker T, Ohlraun S, Otte AC, Tullius M, Kovalenko S, Bogaert AV, Maier W, Rietschel M, Propping P, Nothen MM, Cichon S (2004) Examination of G72 and D-amino-acid oxidase as genetic risk factors for schizophrenia and bipolar affective disorder. Mol Psychiatry 9:203-207

20. Shi J, Badner JA, Gershon ES, Liu C (2008) Allelic association of G72/G30 with schizophrenia and bipolar disorder: a comprehensive meta-analysis. Schizophr Res 98:89-97

21. Wood SJ, Berger G, Velakoulis D, Phillips LJ, McGorry PD, Yung AR, Desmond P, Pantelis C (2003) Proton magnetic resonance spectroscopy in first episode psychosis and ultra high-risk individuals. Schizophr Bull 29:831-843

22. Yung AR, Phillips LJ, McGorry PD, McFarlane CA, Francey S, Harrigan S, Patton GC, Jackson HJ (1998) Prediction of psychosis. A step towards indicated prevention of schizophrenia. Br J Psychiatry Suppl 172:14-20 